\title{
Mit neuem Schwung ins Jahr 2000
}

M it neuem Layout und neuem Schwung geht das Allergo Journal in den neunten Jahrgang. Verlag und Herausgeber haben für das kommende Jahr beschlossen, das erfolgversprechende Konzept mit noch mehr Originalien und Übersichten, mehr Informationen und mehr Schwerpunktthemen fortzuführen. Sie, liebe Leser, möchten wir auffordern, das Allergo Journal durch eigene Beiträge - Originalbeiträge, Übersichten, Leserbriefe, Hinweise aller Art - aktiv zu unterstützen. Ein größerer Umfang des Originaliateils garantiert schnellere Publikation Ihrer Beiträge. Der umfangreiche Rahmenteil informiert darüber hinaus über internationale Literatur, Kongresse und Tagungen. Allergische Krankheiten
stehen im Blickpunkt der
Öffentlichkeit Der Ärzteverband Deutscher Allergologen und die Deutsche Gesellschaft für Allergologie und klinische Immunologie stehen hinter dieser Konzeption und werden alle Verlautbarungen und Positionspapiere auch weiterhin im Allergo Journal veröffentlichen.

Allergische Krankheiten stehen an der Schwelle zum dritten Jahrtausend stärker im Blickpunkt der Öffentlichkeit als je zuvor. Die epidemische $\mathrm{Zu}-$ nahme allergischer Krankheiten in den Industrieländern ist ominöses Signum der vorab nicht absehbaren Konsequenzen veränderter Lebensbedingungen und Symptom unseres mangelnden Verständnisses für den Zusammenhang zwischen Umweltveränderungen und humaner Immunbiologie.

Die neunziger Jahre boten nach der Wiedervereinigung von Ost- und West-Deutschland unwiederbringliche Gelegenheiten zum Studium dieser $\mathrm{Zu}$ sammenhänge, die nicht nur für die deutschsprachige allergologische Epidemiologie einen Quantensprung an internationalem Ansehen brachte, sondern auch tiefgreifende Einsichten in die Abhängigkeit der Entstehung aller- gischer Erkrankungen von den Lebensbedingungen.

Diese Erkenntnisse müssen im kommenden Jahrzehnt vertieft werden, um über epidemiologische Zusammenhänge hinaus kausale Beziehungen zu sichern. Raum ist für Forschungsansätze aller Art und es ist zu hoffen, dass sich hieraus möglichst rasch konkrete Schritte zur Verringerung der Prävalenz allergischer Erkrankungen ergeben werden. Das Spektrum der Ansätze zur Verringerung allergischer Erkrankungen reicht von der Allergenprävention auf breiter Basis (Wohnungsbau, Ernährung, etc.) bis zur präventiven Spezifischen Immuntherapie vor dem Auftreten gravierender Symptome bzw. medikamentöser Therapie.

Die Zeitschrift Nature hat die Bedeutung von Allergie und Asthma durch ein jüngst erschienenes Supplementheft (25.11.1999, Bd. 402, Nr. 6760 www.nature.com) betont. Die Beiträge spannen einen Bogen von den zunehmenden Kenntnissen über die genetischen Grundlagen allergischer Erkrankungen, den Interaktionen zwischen Allergenexposition und viralen bzw. bakteriellen Infektionen bis zu den zahlreichen therapeutischen Ansatzpunkten. Insbesondere wird deutlich, dass sich das

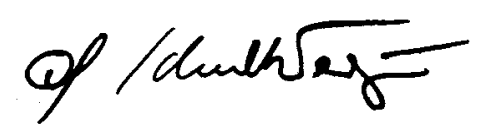

Prof. Dr. G. Schultze-Werninghaus
Interesse an den immunologischen Grundlagen allergischer Erkrankungen zunehmend auf die intrauterine fetale Entwicklung fokussiert. Von besonderem Interesse ist die Interaktion von Infektionen durch Viren und Bakterien mit der Entstehung allergischer Er-

krankungen. Lesenswert hierzu auch die Editorials von Bengt Björkstén, Randolf Brehler und Thomas A. Luger in Heft 6, Bd. 104

(1999) des Journal of Allergy and Clinical Immunology.

Im Gegensatz zu den faszinierenden Fortschritten unserer Kenntnisse über die Grundlagen allergischer Erkrankungen sind seit Jahrzehnten keine grundsätzlich neuen therapeutischen Ansätze zu vermerken. Mit Spannung wird in diesem Zusammenhang die bevorstehende Einführung des AntiImmunglobulin $\mathrm{E}$ erwartet, dessen Konsequenzen für die Verringerung der Prävalenz und der Schwere allergischer Krankheiten noch nicht absehbar sind. Sicher ist bereits jetzt, dass seit der Einführung der Glukokortikosteroide vor 50 Jahren keine therapeutische Entwicklung von einer derartigen Bedeutung gewesen ist.

Herausgeber und Verlag wünschen den Lesern des Allergo Journals ein erfolgreiches Jahr 2000.

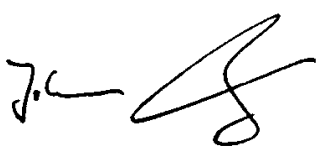

Prof. Dr. Dr. J. Ring 